\title{
PDCD4 expression in coronary atherosclerosis rat models and its mechanism
}

\author{
YUHONG GAO ${ }^{1 *}$, HONGMEI LI ${ }^{1 *}$, YANCHUN ZHOU ${ }^{2}, \mathrm{HONGMEI} \mathrm{LV}^{3}$ and YANPING CHEN ${ }^{4}$ \\ Departments of ${ }^{1}$ Cardiology, ${ }^{2}$ Psychology and ${ }^{3}$ Cardiovascular Medicine, Yidu Central Hospital \\ of Weifang, Weifang, Shandong 262500; ${ }^{4}$ Department of Cardiovascular Medicine, \\ Weifang People's Hospital, Weifang, Shandong 261041, P.R. China
}

Received March 30, 2018; Accepted February 6, 2019

DOI: $10.3892 / \mathrm{etm} .2019 .7296$

\begin{abstract}
This study investigated the expression of programmed cell death protein 4 (PDCD4) in rat models of coronary atherosclerosis (AS) and analyzed its role and mechanism. A total of $80 \mathrm{Wistar}$ rats were selected and divided into the control group $(n=40)$ and research group $(n=40)$ according to the principle of similar body weight, of which coronary AS models were established in rats in the research group. PDCD4 expression in coronary artery tissues was detected using western blotting, and the expression of interleukin-6 (IL-6) and IL- 8 in the coronary artery tissues were measured by means of reverse transcription-polymerase chain reaction (RT-PCR). The apoptotic rate of coronary artery smooth muscle cells was determined via terminal deoxynucleotidyl transferase-mediated dUTP nick end labeling (TUNEL). The relative expression of PDCD4 in coronary artery tissues in the research group was obviously higher than that in the control group, and the difference was statistically significant $(\mathrm{t}=6.121, \mathrm{P}<0.01)$. In terms of the relative expression of messenger ribonucleic acid (mRNA) of IL-6 in the coronary artery tissues, the research group had a remarkably higher level than the control group, with a statistically significant difference $(\mathrm{t}=21.03, \mathrm{P}<0.01)$. The difference in the relative expression of IL- 8 mRNA between the research group and the control group was statistically significant, of which a much higher level was detected in the research group $(\mathrm{t}=19.96, \mathrm{P}<0.01)$. The apoptotic rate of smooth muscle cells in the research group was increased notably compared with that in the control group, and the difference was statistically significant $(\mathrm{t}=5.985, \mathrm{P}<0.01)$. PDCD4 may participate in the formation of coronary AS plaque, and its possible function in
\end{abstract}

Correspondence to: Dr Yanping Chen, Department of Cardiovascular Medicine, Weifang People's Hospital, 151 Guangwen Street, Weifang, Shandong 261041, P.R. China

E-mail:pym53u@163.com

*Contributed equally

Key words: PDCD4, coronary atherosclerosis, IL-6, IL-8, rat models the process is to inhibit the proliferation of vascular smooth muscle cells and promote the upregulation of IL-6 and IL-8.

\section{Introduction}

Acute coronary syndrome (ACS) is a severe type of coronary heart disease, which is a pathological symptom caused by obstructed coronary arteries. It occurs primarily in older people and is a leading cause of poor prognosis and sudden death of patients with coronary heart disease. ACS patients often die suddenly without any sign, so it causes great harms (1). As a common type of cardiovascular system disease, atherosclerosis (AS) is a chronic inflammatory disease of the arterial wall induced by lipid metabolism disorder in the body (2). AS is the major pathological basis of ACS which is a clinical pathological syndrome resulting from total and non-total occlusive thrombus secondary to coronary AS plaque rupture or invasion. Vascular inflammation reactions triggered by inflammatory factors are the main reason of ACS occurrence, which destroys the stability of the coronary AS plaque and causes rupture and hemorrhage of the plaque, thus leading to thrombus $(3,4)$.

The pathogenesis of coronary AS has not been clarified thus far, and studies have demonstrated that both immune cells and inflammatory factors are involved in the process of coronary AS formation (5). Programmed cell death protein 4 (PDCD4) is a novel suppressor gene discovered in recent years, which cannot only control the proliferation of tumor cells but also suppress the generation of tumor cells, having a close correlation with the occurrence and development of tumors (6). According to the study by Liang et al (7), PDCD4 is expressed in myocardial cells and vascular smooth muscle cells, and it can inhibit the expression of the inflammatory factor interleukin-10 (IL-10) by activating nuclear factorkappa $\mathrm{B}(\mathrm{NF}-\kappa \mathrm{B})$ in vascular smooth muscle cells. In addition to its inhibitory effects in the occurrence and development of multiple tumors, PDCD4 also participates in immune response, inflammatory reaction and other pathophysiological processes.

Research on PDCD4 in recent years was mainly focused on the mechanism of tumors, but there are rare studies on its role in coronary AS. This study aimed to analyze the function and mechanism of PDCD4 in the process of coronary AS 
Table I. Primer sequences for IL-6, IL-8 and $\beta$-actin genes.

\begin{tabular}{lll}
\hline Genes & Forward primer sequence & Reverse primer sequence \\
\hline$I L-6$ & 5'-CTCTCCGCAAGAGACTTCCA-3' & 5'-TGGTCTTCTGGAGTTCCGTT-3' \\
$I L-8$ & 5'-CTTTGTCCATTCCCACTTCTGA-3' & 5'-TCCCTAACGGTTGCCTTTGTAT-3' \\
$\beta$-actin & 5'- TGAAGTGTGACGTGGACATC-3' & 5'-TAGAAGCATTTGCGGTGGAC-3'
\end{tabular}

IL-6, interleukin-6.

formation by means of observing the PDCD4 expression in coronary AS plaque of rats.

\section{Materials and methods}

Laboratory animals. A total of 80 healthy, clean and specific pathogen-free (SPF) Wistar rats, aged 6-8 weeks, with a body mass of 170-190 g, were purchased from Shanghai Jia Ke Biotechnology Co., Ltd. (Shanghai, China) [animal certification no. SCXK(Shanghai)2016-18]. The rats were maintained in a clean environment, with indoor temperature of $21-25^{\circ} \mathrm{C}$ and humidity of $52-57 \%$. All the rats were fed adaptively for 2 weeks prior to the experiment. This animal experiment was approved by the Ethics Committee of Yidu Central Hospital of Weifang (Weifang, China).

Main instruments and reagents. Rabbit anti-rat PDCD4 and glyceraldehyde- 3-phosphate dehydrogenase (GAPDH) monoclonal antibodies were purchased from Cell Signaling Technology, Inc. (cat nos. 9535 and 2118; Danvers, MA, USA), bicinchoninic acid (BCA) protein assay kit was obtained from Beijing Solarbio Science \& Technology Co., Ltd. (Beijing, China), and terminal deoxynucleotidyl transferase-mediated dUTP nick end-labeling (TUNEL) apoptosis assay kit was purchased from Beijing Jiamay Biotech Co., Ltd. (Beijing, China). Real-time quantitative polymerase chain reaction (PCR) instrument as well as real-time quantitative PCR kits for interleukin-6 (IL-6) and IL-8 were purchased from Shanghai HuaGen Biotech Co., Ltd. (Shanghai, China). Total ribonucleic acid (RNA) extraction kit (TRIzol reagent method) was obtained from Thermo Fisher Scientific Inc. (Waltham, MA, USA). Promega reverse transcription kit was from ABclonal Biotech Co., Ltd. (Woburn, MA, USA) and the internal reference primers for IL- 6, IL- 8 and $\beta$-actin in reverse-transcription quantitative PCR were purchased from Shanghai Gefan Biotechnology Co., Ltd. (Shanghai, China). Primer sequences are listed in Table I.

Construction and grouping of animal models. The healthy SPF Wistar rats with similar body weight were selected and divided into control group $(n=40)$ and research group $(n=40)$, and rats in the control group were fed with normal diet. By reference to the modeling methods in the literature by Ganzetti et al (8), the rats in the research group were injected with vitamin D3 from the right lower extremity and raised with high-fat diet (recipe: $0.2 \%$ propylthiouracil, $10 \%$ lard, $1.5 \%$ sodium cholate, $4 \%$ cholesterol and $84.3 \%$ basic diet) provided by Guangzhou SeBiona Bio-Tech Co., Ltd. at 30 days before the modeling. At 3 and 6 weeks of feeding, $10 \%$ bovine serum albumin $(250 \mathrm{mg} / \mathrm{kg})$ was injected into the rats from the tail veins for immune damage. At 15 weeks after feeding, the rats were sacrificed by decapitation. In the research group, the tissues of coronary plaque were extracted to examine the cardiac pathology, and the existence of AS plaque suggested successful model establishment. The harvested coronary artery tissues of all the rats were placed into liquid nitrogen immediately and then stored in a refrigerator at $-80^{\circ} \mathrm{C}$.

\section{Index detection}

PDCD4 detection. Western blotting was utilized to measure the PDCD4 in the coronary arteries. The coronary artery tissues were taken and fully ground into tissue homogenates, followed by centrifugation at $3,000 \times \mathrm{g}$ at $4^{\circ} \mathrm{C}$ for $8 \mathrm{~min}$ and preparation of $50 \mu \mathrm{g}$ protein extracts. Next, proteins with $8 \%$ loading sample were extracted for polyacrylamide gel electrophoresis experiment, and were transferred to a polyvinylidene fluoride (PVDF) membrane at the end of electrophoresis. Then the proteins were blocked in 5\% skim milk powder for $1 \mathrm{~h}$, followed by incubation with PDCD4 primary antibody $(1: 1,000)$ and GAPDH polyclonal antibody $(1: 1,000)$ at $4^{\circ} \mathrm{C}$ overnight. Subsequently, goat anti-rabbit horseradish peroxidase (HRP)-labeled secondary polyclonal antibody $(1: 1,000$; cat. no. 7074; Cell Signaling Technology, Inc.) was added and reacted at $37^{\circ} \mathrm{C}$ for $1.5 \mathrm{~h}$, and diaminobenzidine was used for staining and development. Images were captured and stored for analysis and processing. The gel analysis software Quantity One 4.6.2 (Bio-Rad Laboratories, Inc., Hercules, CA, USA) was applied to measure the relative expression of PDCD4.

Detection of IL-6 and IL-8. Reverse transcription PCR (RT-PCR) was performed to detect IL-6 and IL-8 in the coronary arteries, of which the primer and probe sequences are shown in Table I. Experimental procedures involved use of phenol method (TRIzol reagent method) to extract the total RNA in the coronary artery tissues in strict accordance with the TRIzol kit instructions. The concentration and purity of the total RNA were measured using a micro-spectrophotometer (Bio-Rad Laboratories, Inc.), and the integrity was detected by virtue of denaturing agarose gel electrophoresis. The RNA was reverse transcribed into complementary deoxyribonucleic acid (cDNA) via the Promega reverse transcription kit strictly according to the steps in the instructions. The PCR system was prepared as described in the instructions. Reaction conditions were: 35 cycles of pre-denaturation at $94^{\circ} \mathrm{C}$ for $10 \mathrm{~min}$, denaturation at $94^{\circ} \mathrm{C}$ for $30 \mathrm{sec}$, annealing at $60^{\circ} \mathrm{C}$ for $45 \mathrm{sec}$, extension at $72^{\circ} \mathrm{C}$ for $45 \mathrm{sec}$ and final extension at $65^{\circ} \mathrm{C}$ for 
Table II. General information of rats in the two groups.

\begin{tabular}{lcccc}
\hline & $\begin{array}{c}\text { Control } \\
\text { group } \\
(\mathrm{n}=40)\end{array}$ & $\begin{array}{c}\text { Research } \\
\text { group } \\
(\mathrm{n}=36)\end{array}$ & $\mathrm{t} / \chi^{2}$ & P-value \\
Category & & & 0.038 & 0.846 \\
\hline $\begin{array}{l}\text { Sex }(\%) \\
\quad \text { Male }\end{array}$ & $22(55.00)$ & $19(52.78)$ & & \\
$\quad$ Female & $18(45.00)$ & $17(47.22)$ & & \\
$\begin{array}{l}\text { Age (weeks) } \\
\leq 8\end{array}$ & $19(47.50)$ & $15(41.67)$ & & \\
$>8$ & $21(52.50)$ & $21(58.33)$ & & \\
$\begin{array}{l}\text { Body mass }(\mathrm{g}) \\
\quad \leq 180\end{array}$ & $21(52.50)$ & $22(61.11)$ & & \\
$>180$ & $19(47.50)$ & $14(38.89)$ & & \\
$\begin{array}{l}\text { Indoor } \\
\text { temperature }\left({ }^{\circ} \mathrm{C}\right)\end{array}$ & $23.12 \pm 1.63$ & $22.89 \pm 0.98$ & 0.735 & 0.464 \\
$\begin{array}{l}\text { Indoor } \\
\text { humidity }(\%)\end{array}$ & $54.45 \pm 1.03$ & $54.16 \pm 1.37$ & 1.049 & 0.297 \\
\hline
\end{tabular}

$10 \mathrm{~min}$. The software offered by manufacturers was applied to analyze the amplification data, and the relative expression of internal references of IL-6, IL- 8 and $\beta$-actin were calculated by using the formula of $2^{-\Delta \Delta \mathrm{Cq}}(9)$.

Apoptosis rate of coronary artery smooth muscle cells. A portion of coronary artery tissues was fixed in $4 \%$ formaldehyde solution and then embedded in paraffin. After dewaxing and rehydration, TUNEL assay was conducted to detect the apoptosis rate of smooth muscle cells in the coronary artery in strict accordance with the instructions of TUNEL assay kit. Five consecutive high-power fields were examined using a light microscope (Olympus, Tokyo, Japan), and the apoptotic cells were counted in every 100 cells, to calculate the apoptosis rate as per the formula: (Number of apoptotic cells/total number of cells) $\mathrm{x} 100 \%=$ apoptosis rate.

Statistical analysis. Statistical Product and Service Solutions (SPSS) 17.0 software (Tianjin Ksoft Tech. Co., Ltd., Kerala, India) was used for statistical analysis. Measurement data were presented as mean \pm standard deviation (SD). The Student's t-test was performed for comparison of measurement data, and the Chi-square test was used for comparison of enumeration data. $\mathrm{P}<0.05$ was considered to indicate a statistically significant difference.

\section{Results}

General conditions of rats in the control group and the research group. In this experiment, coronary AS models were established in the Wistar rats, and all 40 rats in the research group were sacrificed by decapitation at 15 weeks after feeding. There were 36 successful models, with a success rate of $90.00 \%(36 / 40)$. The sex, age and body mass of the rats, indoor temperature and indoor humidity had no impacts on the experiment $(\mathrm{P}>0.05)$ (Table II).

PDCD4 expression in coronary artery tissues of rats in the control group and research group. The relative expression

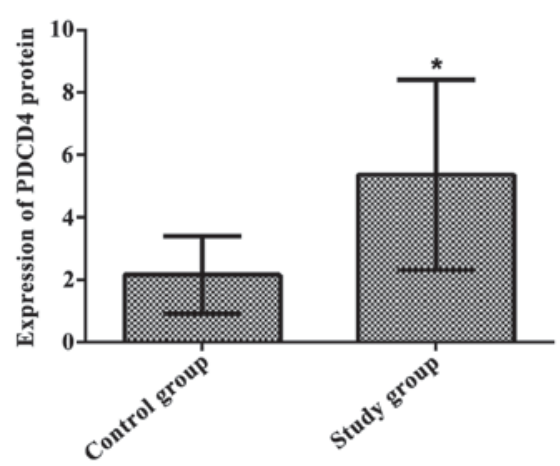

Figure 1. Expression of PDCD4 protein in coronary artery tissue of the control and study groups. Results of western blotting showed that relative expression level of PDCD4 protein in coronary tissue of the study group was significantly higher than that of the control group $(\mathrm{t}=6.121, \mathrm{P}<0.01){ }^{*} \mathrm{P}<0.01$, compared with the control group.

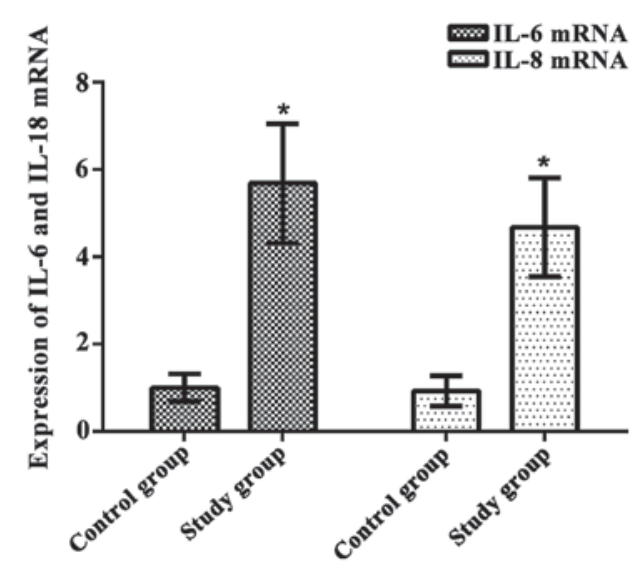

Figure 2. Expression of IL-6 and IL-18 mRNA in coronary artery tissues in the control and study groups. RT-PCR results showed that expression level of IL-6 mRNA in coronary artery tissues of the study group was significantly higher than that of the control group $(\mathrm{t}=21.03, \mathrm{P}<0.01)$. Expression level of IL-8 mRNA in coronary artery tissues of the study group was significantly higher than that of the control group $(\mathrm{t}=19.96, \mathrm{P}<0.01)$. ${ }^{*} \mathrm{P}<0.01$, compared with the control group.

of PDCD4 in coronary artery tissues was $2.16 \pm 1.24$ in the control group and $5.37 \pm 3.05$ in the research group. The relative expression of PDCD4 in coronary artery tissues in the research group was obviously higher than that in the control group, and the difference was statistically significant $(\mathrm{t}=6.121, \mathrm{P}<0.01)$ (Fig. 1).

Messenger RNA (mRNA) expression of IL-6 and IL-8 in coronary artery tissues of rats in the control and research groups. The relative expression of IL- 6 mRNA in coronary artery tissues was $1.01 \pm 0.31$ in the control group, and it was $5.69 \pm 1.37$ in the research group. The research group had a significantly higher level of IL-6 mRNA relative expression than that of the control group, with a statistically significant difference $(\mathrm{t}=21.03, \mathrm{P}<0.01)$. The relative expression of IL-8 mRNA in coronary artery tissues were $0.93 \pm 0.35$ and $4.68 \pm 1.13$ in the control and research groups, respectively. The difference in the relative expression of IL- 8 mRNA between the two groups was statistically significant, and a much higher level was evident in the research group $(\mathrm{t}=19.96, \mathrm{P}<0.01)$ (Fig. 2). 
Table III. Comparison of apoptosis rates of smooth muscle cells between the two groups (mean $\pm \mathrm{SD}$ ).

\begin{tabular}{lcc}
\hline Group & $\mathrm{n}$ & Apoptosis rate $(\%)$ \\
\hline Control group & 40 & $12.56 \pm 9.63$ \\
Research group & 36 & $28.36 \pm 13.26$ \\
$\mathrm{t}$ & & 5.985 \\
P-value & & $<0.001$ \\
\hline
\end{tabular}

Apoptotic rates of smooth muscle cells in the control and research groups. The apoptosis rate of smooth muscle cells was $12.56 \pm 9.63 \%$ in the control group and $28.36 \pm 13.26 \%$ in the research group. The apoptosis rate of smooth muscle cells in the research group was increased notably compared with that in the control group, and the difference was statistically significant $(\mathrm{t}=5.985, \mathrm{P}<0.001)$ (Table III).

\section{Discussion}

Approximately 18 million individuals succumb to atherosclerotic cardiovascular diseases annually worldwide, and ACS is the primary cause of sudden cardiac death of patients. ACS is mainly a consequence of coronary occlusion caused by AS plaque rupture and thrombosis (10). Therefore, AS is a non-linear process alternating between stable phase and unstable phase, and the vulnerability of AS plaque is an initiating agent of ACS (11). Current treatments of AS tend to eliminate the plaques and control vulnerable ones (12). The rupture of unstable AS plaques is one of the causes for ACS, and inflammatory responses are vital factors for the rupture of AS plaques and thrombosis (13). Several theories of the AS pathogenesis have been put forward successively, of which the inflammation theory was the most acceptable, suggesting that inflammatory response plays an important role in various stages of an AS event (14).

As a kind of apoptosis-related gene, $P D C D 4$ can bind to eukaryotic initiation factor-4A (eIF4A) via the functional domain of MA3 protein and repress the combination of eIF4C with eIF4A, thus suppressing the synthesis of ribosome and protein (15). A study indicated that PDCD4 is involved in the reaction of pro-inflammatory Toll-like receptor 4 (TLR4) signaling pathway induced by lipopolysaccharide (LPS); thus, PDCD4 is also considered as a pro-inflammatory protein that may have functions in inflammatory diseases (16). It has been proven in recent years that IL- 6 and IL- 8 are cytokines closely associated with the pathological progression of ACS. IL-6 is a category of inflammatory factors capable of promoting the proliferation and differentiation of B lymphocytes, which participates in the reaction processes of multiple inflammatory diseases. IL-6 can act as an inflammatory marker for local and peripheral blood circulations of AS. In addition, its expression level may reflect the degree of instability of AS plaque (17). IL-8, one of the cytokines with the highest chemotactic activity, can accelerate local thrombosis around the AS plaque and enhance a series of mechanisms of the AS plaque, such as local oxidative stress response (18). The early pathological changes of AS involve in a series of reactions, including changes in endothelial function and aggregation of monocytes and T cells. Moreover, with the progression of the disease, the vascular smooth muscle cells are getting involved. The proliferation and apoptosis always play a crucial role in the formation of plaques (19). Apoptosis of vascular smooth muscle cells participates in the pathological processes of plaque and thrombus formation. Moreover, it can lead to release of various inflammatory factors, including IL-1, IL-6 and IL-8, thus aggravating inflammation symptoms of the AS plaque (20). Research by Green et al (21) manifested that PDCD4 is downregulated remarkably in the arterial smooth muscle cells of rats with acute balloon injury, thus facilitating the proliferation of these cells. Yu and $\mathrm{Li}$ (22) argued that high PDCD4 expression can inhibit the proliferation of vascular smooth muscle cells. Apoptosis of vascular smooth muscle cells is significantly decreased in PDCD4 knockout rats, while the highly expressed PDCD4 can increase such apoptosis notably. Therefore, PDCD4 can regulate the proliferation and apoptosis of smooth muscle cells. However, the results of this research indicated that the relative expressions of PDCD4, IL-6 and IL-8 in coronary artery tissues in the research group were elevated remarkably compared with those in the control group, suggesting that PDCD4 may participate in the formation of AS plaque. The research group had a significantly higher apoptosis rate of smooth muscle cells than that of the control group, and it was considered through further analysis that PDCD4 may increase the expression of inflammatory factors and then upregulate the IL-6 and IL-8 expression by means of suppressing the proliferation of vascular smooth muscle cells during the formation of AS plaque.

Considering the repeatability and reliability of animal experiment, the rats in this study were screened strictly to control the differences in age, body mass, health and other aspects of the Wistar rats. It was shown that the sex, age and body mass of the rats, indoor temperature and humidity had no impacts on the experiment. Since PDCD4 knockout was not performed for the rats and the expression of inflammatory factors in PDCD4 knockout rats were not analyzed, there were certain limitations in this experiment. Therefore, it is expected that the experiment should be conducted in the rats next time, so as to provide more evidence for these findings.

In conclusion, PDCD4 may participate in the formation of coronary AS plaque, and its possible function in the process is to inhibit the proliferation of vascular smooth muscle cells and promote the upregulation of IL-6 and IL-8.

\section{Acknowledgements}

Not applicable.

\section{Funding}

No funding was received.

\section{Availability of data and materials}

The datasets used and/or analyzed during the present study are available from the corresponding author on reasonable request. 


\section{Authors' contributions}

YG and HLi wrote the manuscript and assisted in the construction and grouping of animal models. YZ and HLv performed western blotting and RT-PCR. YC was responsible for TUNEL assay. All authors read and approved the final manuscript.

\section{Ethics approval and consent to participate}

The study was approved by the Ethics Committee of Yidu Central Hospital of Weifang (Weifang, China).

\section{Patient consent for publication}

Not applicable.

\section{Competing interests}

The authors declare that they have no competing interests.

\section{References}

1. Mega JL, Braunwald E, Wiviott SD, Bassand JP, Bhatt DL, Bode C, Burton P, Cohen M, Cook-Bruns N, Fox KA, et al; ATLAS ACS 2-TIMI 51 Investigators: Rivaroxaban in patients with a recent acute coronary syndrome. N Engl J Med 366: 9-19, 2012.

2. Aronow HD and Beckman JA: Parsing atherosclerosis: The unnatural history of peripheral artery disease. Circulation 134: 438-440, 2016

3. Lee T, Murai T, Isobe M and Kakuta T: Impact of coronary plaque morphology assessed by optical coherence tomography on cardiac troponin elevation in patients with non-ST segment elevation acute coronary syndrome. Catheter Cardiovasc Interv 90: 905-914, 2017.

4. De Ronde M, Kok MGM, Beijk MAM, De Winter RJ, Van Der Wal AC, Sondermeijer BM, Meijers JCM, Creemers EE and Pinto-Sietsma SJ: Tissue-derived circulating miRNAs can identify atherosclerosis and plaque instability. Atherosclerosis 263: e37, 2017.

5. Barbato E, Toth GG, Johnson NP, Pijls NH, Fearon WF, Tonino PA, Curzen N, Piroth Z, Rioufol G, Jüni P, et al: A prospective natural history study of coronary atherosclerosis using fractional flow reserve. J Am Coll Cardiol 68: 2247-2255, 2016.

6. Jo SH, Kim DE, Clocchiatti A and Dotto GP: PDCD4 is a CSL associated protein with a transcription repressive function in cancer associated fibroblast activation. Oncotarget 7: 58717-58727, 2016.

7. Liang X, Xu Z, Yuan M, Zhang Y, Zhao B, Wang J, Zhang A and Li G: MicroRNA-16 suppresses the activation of inflammatory macrophages in atherosclerosis by targeting PDCD4. Int J Mol Med 37: 967-975, 2016.

8. Ganzetti GS, Busnelli M, Parolini C, Manzini S, Dellera F, Sirtori CR and Chiesa G: ApoA-I depletion in chow-fed ApoEKO mice severely worsens coronary atherosclerosis development. Atherosclerosis 252: e104, 2016.

9. Livak KJ and Schmittgen TD: Analysis of relative gene expression data using real-time quantitative PCR and the 2(-Delta Delta C(T)) method. Methods 25: 402-408, 2001.
10. Rodriguez F, Maron DJ, Knowles JW, Virani SS, Lin S and Heidenreich PA: Association between intensity of statin therapy and mortality in patients with atherosclerotic cardiovascular disease. JAMA Cardiol 2: 47-54, 2017.

11. Wierer M, Prestel M, Schiller HB, Yan G, Schaab C, Azghandi S, Werner J, Kessler T, Malik R, Murgia M, et al: Compartmentresolved proteomic analysis of mouse aorta during atherosclerotic plaque formation reveals osteoclast-specific protein expression. Mol Cell Proteomics 17: 321-334, 2018.

12. Vogel ME, Idelman G, Konaniah ES and Zucker SD: Bilirubin prevents atherosclerotic lesion formation in low-density lipoprotein receptor-deficient mice by inhibiting endothelial VCAM-1 and ICAM-1 signaling. J Am Heart Assoc 6: 6, 2017.

13. Tsivgoulis G, Katsanos AH, Giannopoulos G, Panagopoulou V, Jatuzis D, Lemnens R, Deftereos S and Kelly PJ: The role of colchicine in the prevention of cerebrovascular ischemia. Curr Pharm Des 24: 668-674, 2018.

14. Kurdi A, De MG and Martinet W: Everolimus attenuates atherosclerotic plaque progression, intraplaque neovascularization, myocardial infarction and sudden death in a mouse model of advanced atherosclerosis. Atherosclerosis 263: e59, 2017.

15. Maeda N, Abdullahi A, Beatty B, Dhanani Z and Adegoke OAJ: Depletion of the mRNA translation initiation inhibitor, programmed cell death protein 4 (PDCD4), impairs L6 myotube formation. Physiol Rep 5: e13395, 2017.

16. Wang L, Jiang Y, Song X, Guo C, Zhu F, Wang X, Wang Q, Shi Y, Wang J, Gao F, et al: Pdcd4 deficiency enhances macrophage lipoautophagy and attenuates foam cell formation and atherosclerosis in mice. Cell Death Dis 7: e2055, 2016.

17. Dongze W, Jiang Y and Tam LS: FRI0523 Short-term efficacy and safety of new biological agents targeting the IL-6, IL-12/23 and IL-17 pathways for active psoriatic arthritis: A network meta-analysis of randomised controlled trials. Annals of the Rheumatic Diseases 76: 689, 2017.

18. Gualtero DF, Viafara-Garcia SM, Morantes SJ, Buitrago DM, Gonzalez OA and Lafaurie GI: Rosuvastatin inhibits interleukin (IL)-8 and IL-6 production in human coronary artery endothelial cells stimulated with Aggregatibacter actinomycetemcomitans serotype b. J Periodontol 88: 225-235, 2017.

19. Shankman LS, Gomez D, Cherepanova OA, Salmon M, Alencar GF, Haskins RM, Swiatlowska P, Newman AA, Greene ES, Straub AC, et al: Corrigendum: KLF4-dependent phenotypic modulation of smooth muscle cells has a key role in atherosclerotic plaque pathogenesis. Nat Med 22: 217, 2016.

20. Fakhry M, Roszkowska M, Briolay A, Bougault C, Guignandon A, Diaz-Hernandez JI, Diaz-Hernandez M, Pikula S, Buchet R, Hamade E, et al: TNAP stimulates vascular smooth muscle cell trans-differentiation into chondrocytes through calcium deposition and BMP-2 activation: Possible implication in atherosclerotic plaque stability. Biochim Biophys Acta 1863: 643-653, 2017.

21. Green DE, Murphy T C and Hart CM: MicroRNA-21 and PDCD4 mediate antiproliferative effects of PPAR in hypoxiaexposed human pulmonary artery smooth muscle cells. Am J Respir Crit Care Med 193: 7279, 2016.

22. Yu X and Li Z: MicroRNAs regulate vascular smooth muscle cell functions in atherosclerosis (Review). Int J Mol Med 34: 923-933, 2014

(i) $($ This work is licensed under a Creative Commons Attribution-NonCommercial-NoDerivatives 4.0 International (CC BY-NC-ND 4.0) License. 